\title{
Cryo-Balloon Catheter Tracking in Atrial Fibrillation Ablation Procedures
}

\author{
Tanja Kurzendorfer ${ }^{1}$, Alexander Brost ${ }^{1}$, Felix Bourier ${ }^{2}$, Martin Koch ${ }^{1}$, Klaus \\ Kurzidim $^{2}$, Joachim Hornegger ${ }^{1}$, and Norbert Strobel $^{3}$ \\ ${ }^{1}$ Pattern Recognition Lab, Friedrich-Alexander-University Erlangen-Nuremberg, \\ Erlangen, Germany \\ ${ }^{2}$ Klinik für Herzrhythmusstörungen, Krankenhaus Barmherzige Brüder, Regensburg, \\ Germany \\ ${ }^{3}$ Siemens AG, Healthcare Sector, Forchheim, Germany \\ Alexander.Brost@cs.fau.de
}

\begin{abstract}
Radio-frequency (RF) catheter ablation has become the standard treatment of atrial fibrillation if pharmacotherapy fails. As an alternative to traditional RF standard ablation catheters, single-shot devices have received more and more interest. One group of these devices are cryo-balloon catheters. Such catheters are designed to electrically isolate a pulmonary vein (PV) with only a few applications, ideally only one. Whereas standard radio-frequency ablation catheters operate point by point, cryo-balloon devices need to be positioned antrally to the pulmonary vein ostium before freezing. If a good seal can be achieved far enough outside of the pulmonary veins, the cryo-balloon is an effective and safe ablation device. The catheters are inserted through a transseptal sheath and are inflated using liquid nitrogen. Single-shot devices, when used successfully, promise a reduction of procedure time and Xray exposure. Single-shot devices based on ablation energies other than $\mathrm{RF}$, may not carry electrodes or electromagnetic sensors. This makes it difficult to visualize them using standard EP mapping systems. As a result, fluoroscopic imaging is needed. Unfortunately, the inflated balloon may be difficult to see under X-ray. To improve this situation, we propose a new method that tracks and enhances the visualization of a cryo-balloon catheter under fluoroscopic imaging. The method involves a 2-D template of the cryo-balloon that is manually initialized and then tracks the balloon device during live X-ray imaging. To improve visualization, a 2-D ellipse is overlaid onto the fluoroscopic imaging to highlight the position of the balloon catheter. The tracking error was calculated as the distance between the tracked catheter template and the manually segmented catheter. Our method achieved 2-D tracking error of $0.60 \mathrm{~mm} \pm 0.32 \mathrm{~mm}$.
\end{abstract}

\section{Introduction}

Radio-frequency (RF) catheter ablation to treat atrial fibrillation (AFib) has now become an accepted treatment option, in particular, when drug therapy fails [1]. 
Catheter ablation procedures are performed in electrophysiology (EP) labs usually equipped with modern C-arm X-ray systems. Augmented fluoroscopy, overlaying 2-D images rendered from either CT, MR, or C-arm CT 3-D data sets onto live fluoroscopic images, can facilitate more precise real-time catheter navigation and also reduce radiation $[2,3]$. As an alternative to regular ablation catheters which operate on a point-wise ablation strategy, single-shot devices have attracted a significant amount of interest. Under ideal conditions, these devices can electrically isolate a pulmonary vein with a single application. One of these devices are cryo-balloon catheters. These catheters are inserted through a trans-septal sheath and can be inflated using liquid nitrogen [4]. Two different catheter types are currently available. They differ only in diameter, which is either $23 \mathrm{~mm}$ or $28 \mathrm{~mm}$. The type of catheter is chosen depending on underlying patient anatomy. Since no mapping system is available yet for localizing cryo-balloon catheters without fluoroscopy, these devices are placed under Xray. Unfortunately, the inflated balloon catheter may be difficult to see using traditional fluoroscopy imaging. Moreover, the diameter of the catheter can only be determined once the balloon is inflated. We propose a method to track and visualize a cryo-balloon device to simplify catheter placement.

\section{Materials and Methods}

The proposed method uses a 2-D template that is manually initialized and then tracked during live X-ray imaging using template matching $[5,6]$. Once the cryoballoon catheter position has been found inside an X-ray image, a 2-D ellipse determined from manual initialization is superimposed onto the live fluoroscopic view to better visualize the position and the dimension of the catheter.

\subsection{Tracking by Template Matching}

On the first frame of the fluoroscopy sequence manual initialization is required to determine a 2-D tracking template. This template is denoted as $\boldsymbol{T} \in \mathbb{R}^{n \times n}$ with $n \in \mathbb{N}$. Denoting the fluoroscopic images as $\boldsymbol{I}_{t} \in \mathbb{R}^{N \times N}$ with $N \in \mathbb{N}$ and $t \in[0, F]$ the number of the frame in the sequence, a pixel of the image can be accessed by using $\boldsymbol{I}_{t}(u, v)$. The same holds for the template. For simplicity, we assume quadratic images to be considered here, but our method was originally designed for coping with non-quadratic images. From manual initialization, the first position of the catheter in the first frame of the sequence $t=0$ is known as $\left(u_{0}, v_{0}\right) \in \mathbb{N}$, with the image axis denoted as $u$ and $v$. This information is used to constrain the search region to be of size $2 M \times 2 M$ with $M \in \mathbb{N}$. To find the catheter in the next frame $t=t+1$, we use a multi-scale grid search and using the sum of squared distances (SSD) as cost function. The best translation in $\mathrm{u}$-direction and $\mathrm{v}$-direction such that the template matches best to the current observed fluoroscopic image is found by solving the following 


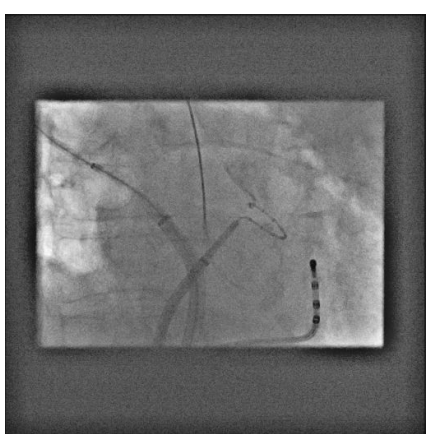

(a)

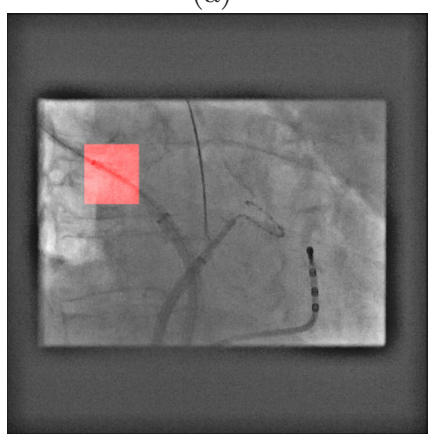

(c)

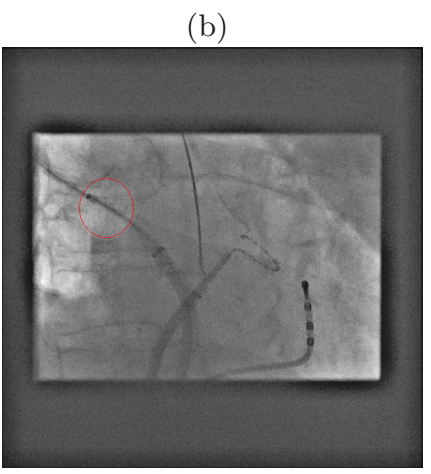

(d)

Fig. 1. (a) First image of one fluoroscopic sequence, $t=0$. (b) Manually initialized 2-D template for tracking. (c) Matched template highlighted in red in the next frame of the sequence, $t=1$. (d) A superimposed ellipse was added to the fluoroscopic image to visualize position and dimensions of the cyro-balloon catheter.

minimization problem

$$
u_{t}, v_{t}=\underbrace{u \in\left[u_{t-1}-M, u_{t-1}+M\right]} \sum_{\substack{i, j \\ \in[-\Delta, \Delta]}}\left(\boldsymbol{I}_{t}(u+i, v+j)-\boldsymbol{T}(i-\Delta, j-\Delta)\right)^{2}
$$

with the half size of the template denoted as $\Delta=\left\lfloor\frac{n-1}{2}\right\rfloor$ and the floor function $\lfloor\cdot\rfloor$ that maps to the largest integer smaller compared to the argument. Our approach is summarized in Fig. 1. The first frame of such a sequence is shown in Fig. 1 (a), the corresponding 2-D template is shown in Fig. 1 (b). The corresponding position in the successive frames is found by finding the best match for the template. The result for one frame is shown in Fig. 1 (c). Finally, the superimposed cryo-balloon position is shown in Fig. 1 (d). 


\section{$3 \quad$ Evaluation and Results}

For the evaluation of the proposed method, 12 clinical sequences were available. The sequences were obtained at one clinical site from 10 patients and were acquired during regular EP procedures on an AXIOM Artis dBC C-arm system (Siemens AG, Forchheim, Germany). Although the data was acquired on a bi-plane system, our catheter tracking approach is not restricted to such a system and will work on a mono-plane device as well. As the sequences were acquired during standard EP procedures, our method is evaluated for a typical setup. It involves one circumferential mapping catheter, one catheter in the coronary sinus and a cryo-balloon catheter. In addition to that, some images show one ECG leads that were attached to the skin of the patient. The tracking error was calculated as the Euclidean distance between the translation vector from the tracking and translation vectors from the manually segmented catheter by a clinical expert. The expert was asked to pick the center of the tip of the cryo-balloon catheter. This is actually not a real tip, but the upper end of the catheter to which the balloon is attached. This upper end is also an opening through which contrast agent can be injected into the pulmonary vein. In contrast to the cryo-balloon itself, the opening can easily be seen as a dark spot in fluoroscopic images. Denoting the gold-standard segmentation by $u_{t}^{\star}$ and $v_{t}^{\star}$, the 2-D error $\varepsilon_{t}$ in $\mathrm{mm}$ is calculated by

$$
\varepsilon_{t}=\rho \cdot \sqrt{\left(\left(u_{t}-u_{t-1}\right)-\left(u_{t}^{\star}-u_{t-1}^{\star}\right)\right)^{2}+\left(\left(v_{t}-v_{t-1}\right)-\left(v_{t}^{\star}-v_{t-1}^{\star}\right)\right)^{2}}
$$

with $t>0$ and the pixel spacing $\rho=0.183 \mathrm{~mm} /$ pixels. The magnification factor was not taken into account. Our proposed method achieved a 2-D tracking error of $0.60 \mathrm{~mm} \pm 0.32 \mathrm{~mm}$ averaged of all frames of all sequences. The results for each sequence are given in Fig. 2. A total minimum error of $0.01 \mathrm{~mm}$ and a total maximum error of $1.64 \mathrm{~mm}$ was found.

\section{Discussion and Conclusions}

Our proposed method successfully tracked a cryo-thermal balloon catheter in 12 clinical sequences. It is able to superimpose the position and diameter of the device onto live fluoroscopic images to enhance the visibility of the cryo-balloon catheter. Manual interaction is only required for the initialization of the template and the determination of the size of the cryo-balloon. After that, the catheter is tracked throughout the remainder of the sequence. The visualized outline of the cryo-balloon helps the physician to see the dimensions of the balloon catheter, otherwise hardly visible under X-ray. Our cryo-balloon catheter tracking method could also be combined with a motion-adjusted 3-D overlay rendered from preoperative data $[7,8]$. Such an example is given in Fig. 3. By doing so, previous balloon catheter positions can be stored and recalled if a second freeze becomes necessary. In addition, a pre-planned cryo-balloon position, e.g., using AFiT [9], could be shown to guide the catheter placement. An example is presented in 


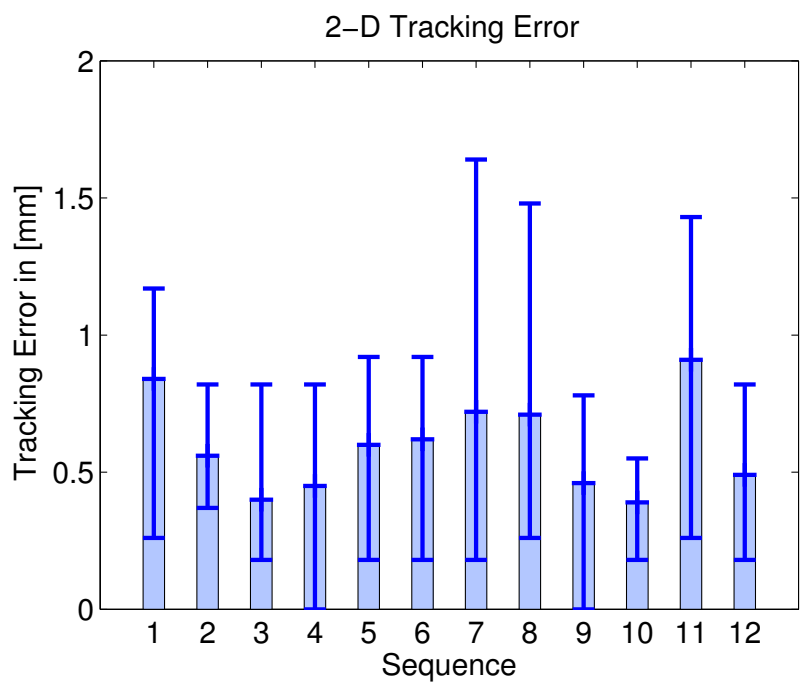

Fig. 2. Two-dimensional catheter tracking error. The proposed method achieves a 2D accuracy of $0.60 \mathrm{~mm} \pm 0.32 \mathrm{~mm}$. A total minimum error of $0.01 \mathrm{~mm}$ and a total maximum error of $1.64 \mathrm{~mm}$ was found.

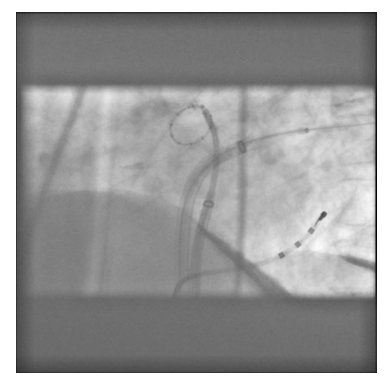

(a)

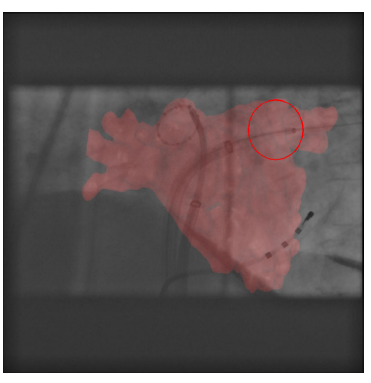

(b)

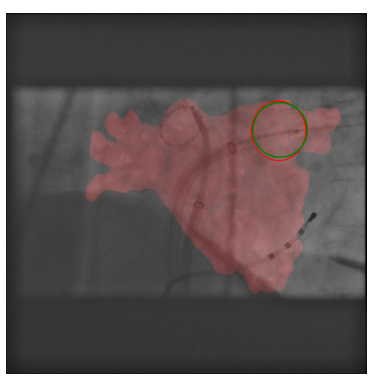

(c)

Fig. 3. (a) Fluoroscopic image of one sequence. (b) The same fluoroscopic image with motion-adjusted overlay and tracked cryo-balloon. (c) The same fluoroscopic image as in (b) with a pre-planned target position of the cryo-balloon (green).

Fig. 3 (c). Future work will focus on tracking the catheter by other means than template matching. Learning based approaches such as in [8] and [10] provide a more robust framework for tracking. In addition, they might also be applicable without manual initialization.

\section{Acknowledgements}

This work has been supported by the German Federal Ministry of Education and Research (BMBF), project grant No. 01EX1012E, in the context of the 
initiative Spitzencluster Medical Valley - Europäische Metropolregion Nürnberg. Additional funding was provided by Siemens AG, Healthcare Sector.

\section{References}

1. Calkins H, Brugada J, Packer D, Cappato R, Chen S, Crijns H, et al. HRS/EHRA/ECAS Expert Consensus Statement on Catheter and Surgical Ablation of Atrial Fibrillation: Recommendations for Personnel, Policy, Procedures and Follow-Up. Europace. 2007 June;9(6):335-379.

2. Sra J, Narayan G, Krum D, Malloy A, Cooley R, Bhatia A, et al. Computed Tomography-Fluoroscopy Image Integration-Guided Catheter Ablation of Atrial Fibrillation. J Cardiovasc Electrophysiol. 2007 April;18(4):409-414.

3. De Buck S, Maes F, Ector J, Bogaert J, Dymarkowski S, Heidbüchel H, et al. An Augmented Reality System for Patient-Specific Guidance of Cardiac Catheter Ablation Procedures. IEEE Transactions on Medical Imaging. 2005 November;24(11):1512-1524.

4. Koller M, Schumacher B. Cryoballoon ablation of paroxysmal atrial fibrillation: bigger is better and simpler is better. Eur Heart J. 2009 Feb;30(6):636-637.

5. Schenderlein M, Rasche V, Dietmayer K. Three-Dimensional Catheter Tip Tracking from Asynchronous Biplane X-Ray Image Sequences using Non-Linear State Filtering. In: Handels H, Ehrhardt J, Deserno TM, Meinzer HP, Tolxdorff T, editors. Bildverarbeitung für die Medizin 2011. Informatik aktuell. Springer Berlin / Heidelberg; 2011. p. $234-238$.

6. Schenderlein M, Dietmayer K. Image-based catheter tip tracking during cardiac ablation therapy. Methods. 2010; p. 1-5.

7. Brost A, Liao R, Strobel N, Hornegger J. Respiratory motion compensation by model-based catheter tracking during EP procedures. Medical Image Analysis. 2010;14(5):695-706. Special Issue on the 12th International Conference on Medical Image Computing and Computer-Assisted Intervention (MICCAI) 2009.

8. Brost A, Wimmer A, Liao R, Hornegger J, Strobel N. Constrained 2-D/3-D Registration for Motion Compensation in AFib Ablation Procedures. In: Taylor R, Yang GZ, editors. Information Processing in Computer-Assisted Interventions. vol. 6689 of Lecture Notes in Computer Science. Springer Berlin / Heidelberg; 2011. p. 133144.

9. Brost A, Bourier F, Kleinoeder A, Raab J, Koch M, Stamminger M, et al. AFiT Atrial Fibrillation Ablation Planning Tool. In: Vision, Modeling, and Visualization (2011); 2011. p. 223-230.

10. Wu W, Chen T, Barbu A, Wang P, Strobel N, Zhou SK, et al. Learning-based hypothesis fusion for robust catheter tracking in 2D X-ray fluoroscopy. In: Computer Vision and Pattern Recognition (CVPR), 2011 IEEE Conference on; 2011. p. 1097 -1104 . 